<smiles>O=C(CCCc1ccc2ccc3cccc4ccc1c2c34)N(C(=O)NC1CCCCC1)C1CCCCC1</smiles>

\title{
Crystal structure of 1,3-dicyclohexyl-1- [3-(pyren-1-yl)propanoyl]urea
}

\section{Edgar González-Juárez, ${ }^{a}$ Marisol Güizado-Rodríguez, ${ }^{a}$ Victor Barba ${ }^{\mathrm{b} *}$ and Hugo Tlahuext ${ }^{\mathrm{b}}$}

${ }^{a}$ Centro de Investigación en Ingeniería y Ciencias Aplicadas, Universidad Autónoma del Estado de Morelos, Av. Universidad No. 1001 Col. Chamilpa, CP 62209, Cuernavaca Mor., Mexico, and ${ }^{\mathbf{b}}$ Centro de Investigaciónes Químicas, Universidad Autónoma del Estado de Morelos, Av. Universidad No. 1001 Col. Chamilpa, CP 62209, Cuernavaca Mor., Mexico. *Correspondence e-mail: vbarba@uaem.mx

\section{Received 5 August 2015; accepted 26 August 2015}

Edited by H. Ishida, Okayama University, Japan

In the title compound, $\mathrm{C}_{33} \mathrm{H}_{38} \mathrm{~N}_{2} \mathrm{O}_{2}$, each of the cyclohexyl rings adopts a chair conformation. The two planes involving carbonyl groups, $\mathrm{C}-(\mathrm{C}=\mathrm{O})-\mathrm{N}$ and $\mathrm{N}-(\mathrm{C}=\mathrm{O})-\mathrm{N}$, are oriented at a dihedral angle of $62.28(10)^{\circ}$. In the crystal, two neighboring molecules are linked by a pair of $\mathrm{N}-\mathrm{H} \cdots \mathrm{O}$ interactions, generating an inversion dimer. The dimers are interconnected by $\mathrm{C}-\mathrm{H}$. . O hydrogen bonds into a supramolecular chain along the $a$-axis direction.

Keywords: crystal structure; $N, N^{\prime}$-dicyclohexylcarbodimide; $N, N^{\prime}$-dicyclohexylurea; hydrogen bonds.

CCDC reference: 1420776

\section{Related literature}

For the synthesis of the title compound, see: Abd-El-Aziz et al. (2013). For the syntheses of $N, N^{\prime}$-dicyclohexylcarbodiimide and $N$-acyl- $N, N^{\prime}$-dicyclohexylurea, see: Zhu et al. (2008); Gonçalves \& Balogh (2006); Kaiser et al. (2008); Slebioda (1995). For related crystal structures, see: Chérioux et al. (2002); Cai et al. (2009); Imhof (2007); Dhinaa et al. (2010); Pinheiro et al. (2011).

\section{Experimental}

\subsection{Crystal data}

$\mathrm{C}_{33} \mathrm{H}_{38} \mathrm{~N}_{2} \mathrm{O}_{2}$

$M_{r}=494.65$

Triclinic, $P \overline{1}$

$a=9.0505(15) \AA$

$b=10.1845(17) \AA$

$c=14.571(2) \AA$

$\alpha=99.541$ (3)

$\beta=90.315(3)^{\circ}$

\subsection{Data collection}

Bruker SMART APEX CCD areadetector diffractometer

Absorption correction: multi-scan (SADABS; Bruker, 2000)

$T_{\text {min }}=0.988, T_{\text {max }}=0.992$

$\gamma=92.191(3)^{\circ}$

$V=1323.4(4) \AA^{3}$

$Z=2$

Mo $K \alpha$ radiation

$\mu=0.08 \mathrm{~mm}^{-1}$

$T=100 \mathrm{~K}$

$0.16 \times 0.13 \times 0.11 \mathrm{~mm}$

\subsection{Refinement}

$R\left[F^{2}>2 \sigma\left(F^{2}\right)\right]=0.052$

$w R\left(F^{2}\right)=0.128$

$S=1.04$

4658 reflections

338 parameters

1 restraint

12906 measured reflections 4658 independent reflections 3738 reflections with $I>2 \sigma(I)$ $R_{\text {int }}=0.050$

Table 1

Hydrogen-bond geometry $\left(\AA,^{\circ}\right)$.

\begin{tabular}{lllll}
\hline$D-\mathrm{H} \cdots A$ & $D-\mathrm{H}$ & $\mathrm{H} \cdots A$ & $D \cdots A$ & $D-\mathrm{H} \cdots A$ \\
\hline $\mathrm{N} 2-\mathrm{H} 2 A \cdots \mathrm{O} 1^{\mathrm{i}}$ & $0.86(1)$ & $2.17(1)$ & $3.026(2)$ & $176(2)$ \\
$\mathrm{C} 2-\mathrm{H} 2 D \cdots \mathrm{O} 2^{\mathrm{ii}}$ & 0.99 & 2.49 & $3.358(2)$ & 146 \\
$\mathrm{C} 4-\mathrm{H} 4 B \cdots \mathrm{O} 2^{\mathrm{ii}}$ & 0.99 & 2.45 & $3.302(2)$ & 144 \\
\hline
\end{tabular}

Symmetry codes: (i) $-x,-y+1,-z+1$; (ii) $-x+1,-y+1,-z+1$.

Data collection: SMART (Bruker, 2000); cell refinement: SAINT (Bruker, 2000); data reduction: $S A I N T$; $\operatorname{program}(\mathrm{s})$ used to solve structure: SHELXS97 (Sheldrick, 2008); program(s) used to refine structure: SHELXL97 (Sheldrick, 2008); molecular graphics: SHELXTL (Sheldrick, 2008); software used to prepare material for publication: SHELXTL, DIAMOND (Brandenburg, 1997), PLATON (Spek, 2009) and publCIF (Westrip, 2010).

\section{Acknowledgements}

Finantial support from CONACYT (grant No. CB2007-81383Q) and for a scholarship to EGJ (grant No. 132946) is gratefully acknowledged. 
Supporting information for this paper is available from the IUCr electronic archives (Reference: IS5410).

\section{References}

Abd-El-Aziz, A. S., Dalgakiran, S., Kucukkaya, I. \& Wagner, B. D. (2013). Electrochim. Acta, 89, 445-453.

Brandenburg, K. (1997). DIAMOND. University of Bonn, Germany.

Bruker (2000). SMART, SAINT and SADABS. Bruker AXS Inc., Madison, Wisconsin, USA.

Cai, X.-Q., Yan, X.-W. \& Xie, X.-N. (2009). Z. Kristallogr. New Cryst. Struct. 224, 211-212.

Chérioux, F., Therrien, B., Stoeckli-Evans, H. \& Süss-Fink, G. (2002). Acta Cryst. E58, o27-o29.
Dhinaa, A. N., Jagan, R., Sivakumar, K. \& Chinnakali, K. (2010). Acta Cryst. E66, o1291.

Gonçalves, V. C. \& Balogh, D. T. (2006). Eur. Polym. J. 42, 3303-3310.

Imhof, W. (2007). Acta Cryst. E63, o4036-o4037.

Kaiser, C. R., Pinheiro, A. C., de Souza, M. V. N., Wardell, J. L. \& Wardell, S. M. S. V. (2008). J. Chem. Res. (S), pp. 468-472.

Pinheiro, A. C., de Souza, M. V. N., Wardell, J. L., Wardell, S. M. S. V. \& Tiekink, E. R. T. (2011). Acta Cryst. E67, o2676-o2677.

Sheldrick, G. M. (2008). Acta Cryst. A64, 112-122.

Ślebioda, M. (1995). Tetrahedron, 51, 7829-7834.

Spek, A. L. (2009). Acta Cryst. D65, 148-155.

Westrip, S. P. (2010). J. Appl. Cryst. 43, 920-925.

Zhu, L., Zhu, Y., Meng, X., Hao, J., Li, Q., Wei, Y. \& Lin, Y. (2008). Chem. Eur. J. 14, 10923-10927. 


\section{supporting information}

Acta Cryst. (2015). E71, o737-o738 [doi:10.1107/S2056989015015996]

\section{Crystal structure of 1,3-dicyclohexyl-1-[3-(pyren-1-yl)propanoyl]urea}

\section{Edgar González-Juárez, Marisol Güizado-Rodríguez, Victor Barba and Hugo Tlahuext}

\section{S1. Comment}

$N, N^{\prime}$-Dicyclohexylcarbodiimide has been used to form esters from carboxylic acid, alcohols and catalytic amounts of 2,6dimethylpyridine (Zhu et al., 2008; Gonçalves \& Balogh, 2006). Nonetheless, the absence of alcohols produce the formation of $N$-acyl- $N, N^{\prime}$-dicyclohexylureas (Kaiser et al., 2008). When arenecarboxylic acids are used, the yield reaction can be modulated by electronic effects of the substituents (Slebioda, 1995). Several crystal structures of $N$-(arenecarbonyl)- $N, N^{\prime}$-dicyclohexylurea derivatives have been reported (Chérioux et al., 2002; Cai et al., 2009; Imhof 2007; Dhinaa et al., 2010; Pinheiro et al., 2011). Herein, we now report the crystal structure of 1,3-dicyclohexyl-1-[(1-pyrenepropyl)carbonyl]urea (I).

In the molecular structure of I, the pyrenyl group and the two planes involving urea nitrogen atoms N1 and N2, $\mathrm{C} 27 / \mathrm{N} 1 / \mathrm{C} 21 / \mathrm{C} 1$ and $\mathrm{C} 28 / \mathrm{N} 2 / \mathrm{C} 27 / \mathrm{H} 2 \mathrm{~A}$, are almost planar with r.m.s. deviations of 0.008 (2), 0.0346 (18) and 0.0098 (18) $\AA$, respectively. The interplanar angle between the $\mathrm{C} 27 / \mathrm{N} 1 / \mathrm{C} 21 / \mathrm{C} 1$ and $\mathrm{C} 28 / \mathrm{N} 2 / \mathrm{C} 27 / \mathrm{H} 2 \mathrm{~A}$ planes is $61.1(6)^{\circ}$. Each of the cyclohexyl rings adopts a chair conformation (Fig. 1). In the crystal, two neighboring molecules are linked by a pair of $\mathrm{N}$ $-\mathrm{H} \cdots \mathrm{O}$ interactions, generating an inversion dimer. The dimers are interconnected by $\mathrm{C}-\mathrm{H} \cdots \mathrm{O}$ hydrogen bonds into a supramolecular chain along the $a$ axis (Fig. 2 and Table 1).

\section{S2. Experimental}

Compound I was obtained according to the literature (Abd-El-Aziz et al., 2013) from an incomplete esterification reaction between pyrenobutanoic acid $(6.80 \mathrm{mmol}), N, N^{\prime}$-dicyclohexylcarbodiimide (7.48 mmol), 2,6-dimethylpyridine $(1.08 \mathrm{mmol})$ as catalyst and 2-(thiophene-3-yl) ethanol $(13.6 \mathrm{mmol})$. The three first components were stirred under room temperature for $1.5 \mathrm{~h}$ using $40 \mathrm{ml}$ of toluene, then the last component was added and heated $1 \mathrm{~h}$ under reflux. (I) was isolated in a yield $c a 8 \%$ from a column chromatography using hexane-ethyl acetate system 4:1. From slow evaporation of the mixture solution, suitable crystals for X-ray diffraction were obtained $\left(m . p .=164{ }^{\circ} \mathrm{C}\right)$.

\section{S3. Refinement}

$\mathrm{H}$ atoms were positioned geometrically $[\mathrm{C}-\mathrm{H}=0.95 \AA$ (aryl), $0.99 \AA$ (methylene) and $1.00 \AA$ (methine) $]$ and constrained using a riding-model approximation with $U_{\mathrm{iso}}(\mathrm{H})=1.2 U_{\mathrm{eq}}(\mathrm{C})$. The $\mathrm{H}$ atom bonded to $\mathrm{N}(\mathrm{H} 2 \mathrm{~A})$ was located in a difference Fourier map and refined freely with an $\mathrm{N}-\mathrm{H}$ distance restraint of 0.86 (1) $\AA$. 


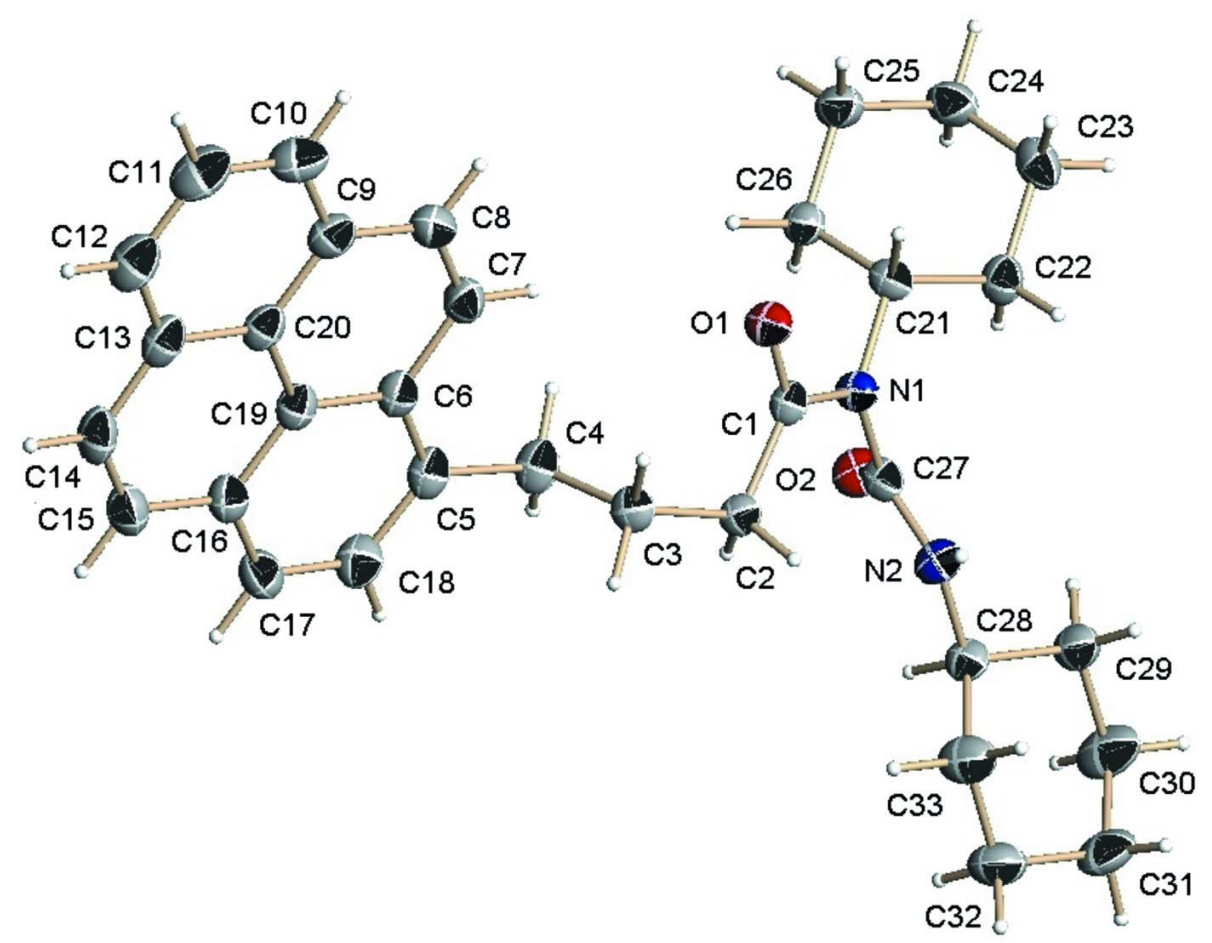

\section{Figure 1}

The molecular structure of the title compound, showing the atom labelling. Displacement ellipsoids are drawn at the $50 \%$ probability level. 


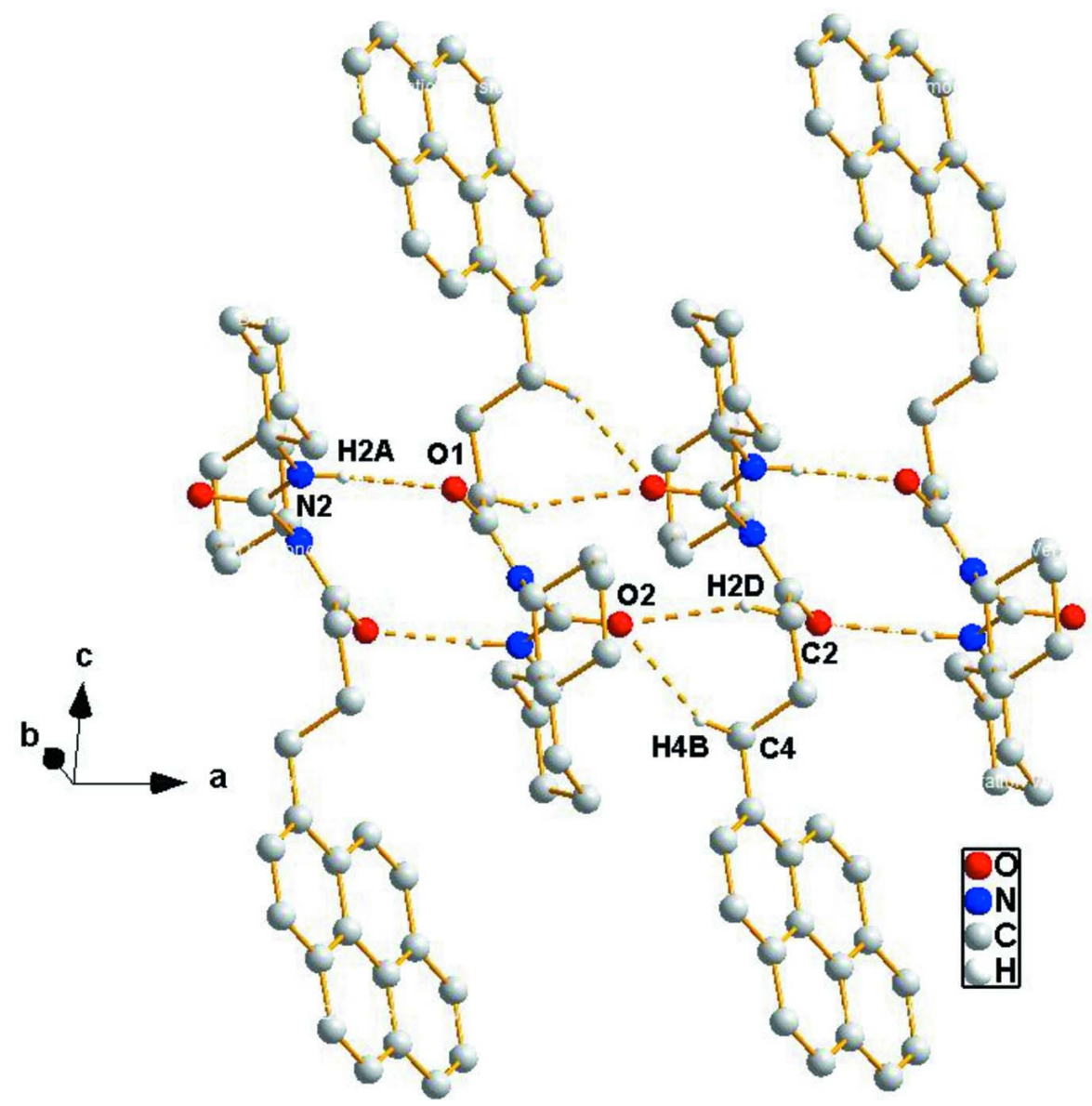

\section{Figure 2}

A view of the crystal packing of the title compound. Hydrogen atoms not involved in the hydrogen bonds (dashed lines) have been omitted for clarity.

\section{1,3-Dicyclohexyl-1-[3-(pyren-1-yl)propanoyl]urea}

\section{Crystal data}

$\mathrm{C}_{33} \mathrm{H}_{38} \mathrm{~N}_{2} \mathrm{O}_{2}$

$M_{r}=494.65$

Triclinic, $P \overline{1}$

Hall symbol: -P 1

$a=9.0505(15) \AA$

$b=10.1845$ (17) $\AA$

$c=14.571(2) \AA$

$\alpha=99.541(3)^{\circ}$

$\beta=90.315(3)^{\circ}$

$\gamma=92.191(3)^{\circ}$

$V=1323.4(4) \AA^{3}$

Data collection

Bruker SMART APEX CCD area-detector diffractometer

Radiation source: fine-focus sealed tube

Graphite monochromator
$Z=2$

$F(000)=532$

$D_{\mathrm{x}}=1.241 \mathrm{Mg} \mathrm{m}^{-3}$

Mo $K \alpha$ radiation, $\lambda=0.71073 \AA$

Cell parameters from 5456 reflections

$\theta=2.6-28.2^{\circ}$

$\mu=0.08 \mathrm{~mm}^{-1}$

$T=100 \mathrm{~K}$

Plates, colourless

$0.16 \times 0.13 \times 0.11 \mathrm{~mm}$

Detector resolution: 8.3 pixels $\mathrm{mm}^{-1}$

phi and $\omega$ scans

Absorption correction: multi-scan

(SADABS; Bruker, 2000) 
$T_{\min }=0.988, T_{\max }=0.992$

12906 measured reflections

4658 independent reflections

3738 reflections with $I>2 \sigma(I)$

$R_{\text {int }}=0.050$

Refinement

Refinement on $F^{2}$

Least-squares matrix: full

$R\left[F^{2}>2 \sigma\left(F^{2}\right)\right]=0.052$

$w R\left(F^{2}\right)=0.128$

$S=1.04$

4658 reflections

338 parameters

1 restraint

Primary atom site location: structure-invariant

direct methods

$$
\begin{aligned}
& \theta_{\max }=25.0^{\circ}, \theta_{\min }=1.4^{\circ} \\
& h=-10 \rightarrow 10 \\
& k=-12 \rightarrow 12 \\
& l=-17 \rightarrow 17
\end{aligned}
$$

\section{Special details}

Experimental. ${ }^{1} \mathrm{H} \mathrm{NMR}\left(400 \mathrm{MHz}, \mathrm{CDCl}_{3}\right) \delta: 8.29(\mathrm{~d}, J=9.2 \mathrm{~Hz}, 2 \mathrm{H}), 8.15(\mathrm{~d}, J=7.8 \mathrm{~Hz}, 2 \mathrm{H}), 8.10(\mathrm{dd}, J=7.7,6.8$ $\mathrm{Hz}, 2 \mathrm{H}), 8.0$ (t, $J=7.7 \mathrm{~Hz}, 1 \mathrm{H}), 7.85(\mathrm{~d}, J=7.8 \mathrm{HZ}, 2 \mathrm{H}), 5.27(\mathrm{~s}, 1 \mathrm{H}), 3.90$ (qn, J = 7.0 Hz, 2H), 3.36 (t, J =7.2 Hz, 2H), $2.38(\mathrm{t}, \mathrm{J}=7.2 \mathrm{~Hz}, 2 \mathrm{H}), 2.18$ (qn, J = 7.2 Hz, 2H), 0.6-1.8 (m, 20H). IR (KBr) $\left(\mathrm{cm}^{-1}\right)=3299(w), 2930(m), 2859(w)$, $1702(s), 1633(s), 1534(m), 1365(m), 1239(m), 835(s)$. EI-MS m/z (\%): $494(M+, 5), 369(50), 228(100), 215(45)$.

Geometry. All e.s.d.'s (except the e.s.d. in the dihedral angle between two l.s. planes) are estimated using the full covariance matrix. The cell e.s.d.'s are taken into account individually in the estimation of e.s.d.'s in distances, angles and torsion angles; correlations between e.s.d.'s in cell parameters are only used when they are defined by crystal symmetry. An approximate (isotropic) treatment of cell e.s.d.'s is used for estimating e.s.d.'s involving 1.s. planes.

Refinement. Refinement of $F^{2}$ against ALL reflections. The weighted $R$-factor $w R$ and goodness of fit $S$ are based on $F^{2}$, conventional $R$-factors $R$ are based on $F$, with $F$ set to zero for negative $F^{2}$. The threshold expression of $F^{2}>\sigma\left(F^{2}\right)$ is used only for calculating $R$-factors(gt) $e t c$. and is not relevant to the choice of reflections for refinement. $R$-factors based on $F^{2}$ are statistically about twice as large as those based on $F$, and $R$-factors based on ALL data will be even larger.

Fractional atomic coordinates and isotropic or equivalent isotropic displacement parameters $\left(\hat{A}^{2}\right)$

\begin{tabular}{lllll}
\hline & $x$ & $y$ & $z$ & $U_{\text {iso }} * / U_{\text {eq }}$ \\
\hline C1 & $0.15199(19)$ & $0.48523(17)$ & $0.56198(11)$ & $0.0247(4)$ \\
C2 & $0.1888(2)$ & $0.63158(17)$ & $0.56245(12)$ & $0.0265(4)$ \\
H2C & 0.1232 & 0.6644 & 0.5173 & $0.032^{*}$ \\
H2D & 0.2920 & 0.6421 & 0.5418 & $0.032^{*}$ \\
C3 & $0.1715(2)$ & $0.71654(18)$ & $0.65839(12)$ & $0.0295(4)$ \\
H3A & 0.1728 & 0.8117 & 0.6516 & $0.035^{*}$ \\
H3B & 0.0743 & 0.6941 & 0.6839 & $0.035^{*}$ \\
C4 & $0.2932(2)$ & $0.6959(2)$ & $0.72692(12)$ & $0.0351(5)$ \\
H4A & 0.2843 & 0.6033 & 0.7394 & $0.042^{*}$ \\
H4B & 0.3906 & 0.7079 & 0.6983 & $0.042^{*}$ \\
C5 & $0.2863(2)$ & $0.79106(19)$ & $0.81785(12)$ & $0.0322(4)$ \\
C6 & $0.1854(2)$ & $0.76932(18)$ & $0.88786(12)$ & $0.0292(4)$ \\
C7 & $0.0873(2)$ & $0.65401(18)$ & $0.87947(13)$ & $0.0336(5)$ \\
H7 & 0.0897 & 0.5889 & 0.8247 & $0.040^{*}$ \\
C8 & $-0.0083(2)$ & $0.63533(19)$ & $0.94717(13)$ & $0.0366(5)$ \\
H8 & -0.0714 & 0.5575 & 0.9387 & $0.044 *$
\end{tabular}




\begin{tabular}{|c|c|c|c|c|}
\hline C9 & $-0.0176(2)$ & $0.72924(19)$ & $1.03139(13)$ & $0.0348(5)$ \\
\hline $\mathrm{C} 10$ & $-0.1161(2)$ & $0.7118(2)$ & $1.10233(15)$ & $0.0450(5)$ \\
\hline H10 & -0.1796 & 0.6342 & 1.0956 & $0.054^{*}$ \\
\hline C11 & $-0.1221(3)$ & $0.8056(2)$ & $1.18188(15)$ & $0.0508(6)$ \\
\hline H11 & -0.1890 & 0.7918 & 1.2297 & $0.061^{*}$ \\
\hline C12 & $-0.0319(2)$ & $0.9196(2)$ & $1.19279(14)$ & $0.0440(5)$ \\
\hline H12 & -0.0386 & 0.9838 & 1.2478 & $0.053^{*}$ \\
\hline $\mathrm{C} 13$ & $0.0690(2)$ & $0.94226(19)$ & $1.12456(12)$ & $0.0346(5)$ \\
\hline $\mathrm{C} 14$ & $0.1642(2)$ & $1.0593(2)$ & $1.13308(13)$ & $0.0392(5)$ \\
\hline H14 & 0.1596 & 1.1249 & 1.1875 & $0.047 *$ \\
\hline C15 & $0.2598(2)$ & $1.0785(2)$ & $1.06597(14)$ & $0.0381(5)$ \\
\hline H15 & 0.3211 & 1.1574 & 1.0739 & $0.046^{*}$ \\
\hline $\mathrm{C} 16$ & $0.2715(2)$ & $0.98252(18)$ & $0.98232(13)$ & $0.0319(4)$ \\
\hline $\mathrm{C} 17$ & $0.3699(2)$ & $1.0003(2)$ & $0.91197(13)$ & $0.0382(5)$ \\
\hline H17 & 0.4333 & 1.0780 & 0.9188 & $0.046^{*}$ \\
\hline $\mathrm{C} 18$ & $0.3764(2)$ & $0.9061(2)$ & $0.83215(13)$ & $0.0383(5)$ \\
\hline H18 & 0.4449 & 0.9207 & 0.7854 & $0.046^{*}$ \\
\hline $\mathrm{C} 19$ & $0.1783(2)$ & $0.86558(18)$ & $0.97074(12)$ & $0.0296(4)$ \\
\hline $\mathrm{C} 20$ & $0.0770(2)$ & $0.84527(18)$ & $1.04232(12)$ & $0.0301(4)$ \\
\hline $\mathrm{C} 21$ & $0.20051(19)$ & $0.25014(17)$ & $0.50327(12)$ & $0.0272(4)$ \\
\hline $\mathrm{H} 21$ & 0.1011 & 0.2347 & 0.5300 & $0.033^{*}$ \\
\hline $\mathrm{C} 22$ & $0.2051(2)$ & $0.16123(18)$ & $0.40814(12)$ & $0.0329(5)$ \\
\hline $\mathrm{H} 22 \mathrm{~A}$ & 0.1279 & 0.1870 & 0.3669 & $0.040^{*}$ \\
\hline $\mathrm{H} 22 \mathrm{~B}$ & 0.3024 & 0.1735 & 0.3794 & $0.040^{*}$ \\
\hline $\mathrm{C} 23$ & $0.1795(2)$ & $0.01573(19)$ & $0.41794(14)$ & $0.0376(5)$ \\
\hline $\mathrm{H} 23 \mathrm{~A}$ & 0.0779 & 0.0019 & 0.4402 & $0.045^{*}$ \\
\hline $\mathrm{H} 23 \mathrm{~B}$ & 0.1890 & -0.0409 & 0.3563 & $0.045^{*}$ \\
\hline $\mathrm{C} 24$ & $0.2896(2)$ & $-0.02592(19)$ & $0.48565(14)$ & $0.0396(5)$ \\
\hline $\mathrm{H} 24 \mathrm{~A}$ & 0.3900 & -0.0238 & 0.4592 & $0.048^{*}$ \\
\hline H24B & 0.2648 & -0.1186 & 0.4943 & $0.048^{*}$ \\
\hline $\mathrm{C} 25$ & $0.2887(2)$ & $0.06563(18)$ & $0.57992(13)$ & $0.0374(5)$ \\
\hline $\mathrm{H} 25 \mathrm{~A}$ & 0.1922 & 0.0548 & 0.6100 & $0.045^{*}$ \\
\hline $\mathrm{H} 25 \mathrm{~B}$ & 0.3668 & 0.0398 & 0.6206 & $0.045^{*}$ \\
\hline $\mathrm{C} 26$ & $0.3151(2)$ & $0.21121(18)$ & $0.56959(12)$ & $0.0305(4)$ \\
\hline $\mathrm{H} 26 \mathrm{~A}$ & 0.3083 & 0.2686 & 0.6312 & $0.037^{*}$ \\
\hline H26B & 0.4156 & 0.2244 & 0.5452 & $0.037^{*}$ \\
\hline $\mathrm{C} 27$ & $0.3174(2)$ & $0.42850(17)$ & $0.42681(12)$ & $0.0264(4)$ \\
\hline $\mathrm{C} 28$ & $0.3480(2)$ & $0.54841(18)$ & $0.29600(12)$ & $0.0290(4)$ \\
\hline $\mathrm{H} 28$ & 0.4519 & 0.5645 & 0.3200 & $0.035^{*}$ \\
\hline $\mathrm{C} 29$ & $0.3498(2)$ & $0.44741(19)$ & $0.20684(12)$ & $0.0368(5)$ \\
\hline $\mathrm{H} 29 \mathrm{~A}$ & 0.2471 & 0.4248 & 0.1844 & $0.044^{*}$ \\
\hline H29B & 0.3940 & 0.3648 & 0.2197 & $0.044 *$ \\
\hline $\mathrm{C} 30$ & $0.4382(3)$ & $0.5017(2)$ & $0.13153(15)$ & $0.0547(6)$ \\
\hline $\mathrm{H} 30 \mathrm{~A}$ & 0.5438 & 0.5123 & 0.1505 & $0.066^{*}$ \\
\hline H30B & 0.4303 & 0.4367 & 0.0729 & $0.066^{*}$ \\
\hline C31 & $0.3839(3)$ & $0.6349(2)$ & $0.11436(14)$ & $0.0485(6)$ \\
\hline H31A & 0.4491 & 0.6700 & 0.0690 & $0.058^{*}$ \\
\hline H31B & 0.2828 & 0.6221 & 0.0872 & $0.058^{*}$ \\
\hline
\end{tabular}


supporting information

$\begin{array}{lllll}\text { C32 } & 0.3822(3) & 0.7348(2) & 0.20321(14) & 0.0459(5) \\ \text { H32A } & 0.3393 & 0.8181 & 0.1906 & 0.055^{*} \\ \text { H32B } & 0.4848 & 0.7562 & 0.2263 & 0.055^{*} \\ \text { C33 } & 0.2913(3) & 0.6800(2) & 0.27775(14) & 0.0450(6) \\ \text { H33A } & 0.2962 & 0.7454 & 0.3362 & 0.054^{*} \\ \text { H33B } & 0.1866 & 0.6673 & 0.2572 & 0.054^{*} \\ \text { H2A } & 0.1664(11) & 0.5120(18) & 0.3687(12) & 0.028(5)^{*} \\ \text { N1 } & 0.21491(16) & 0.39366(14) & 0.49557(10) & 0.0257(3) \\ \text { N2 } & 0.25912(17) & 0.49893(15) & 0.36770(10) & 0.0297(4) \\ \text { O1 } & 0.06756(13) & 0.44841(12) & 0.61964(8) & 0.0314(3) \\ \text { O2 } & 0.44266(14) & 0.38979(13) & 0.42418(9) & 0.0353(3)\end{array}$

Atomic displacement parameters $\left(\AA^{2}\right)$

\begin{tabular}{|c|c|c|c|c|c|c|}
\hline & $U^{11}$ & $U^{22}$ & $U^{33}$ & $U^{12}$ & $U^{13}$ & $U^{23}$ \\
\hline $\mathrm{C} 1$ & $0.0230(9)$ & $0.0294(10)$ & $0.0212(9)$ & $0.0054(7)$ & $0.0028(7)$ & $0.0014(8)$ \\
\hline $\mathrm{C} 2$ & $0.0285(10)$ & $0.0267(10)$ & $0.0242(9)$ & $0.0052(8)$ & $0.0049(7)$ & $0.0023(7)$ \\
\hline $\mathrm{C} 3$ & $0.0317(10)$ & $0.0272(10)$ & $0.0286(10)$ & $0.0072(8)$ & $0.0063(8)$ & $-0.0004(8)$ \\
\hline $\mathrm{C} 4$ & $0.0336(11)$ & $0.0422(12)$ & $0.0277(10)$ & $0.0115(9)$ & $0.0044(8)$ & $-0.0015(9)$ \\
\hline $\mathrm{C} 5$ & $0.0297(10)$ & $0.0386(11)$ & $0.0272(10)$ & $0.0099(9)$ & $0.0001(8)$ & $0.0002(8)$ \\
\hline C6 & $0.0317(10)$ & $0.0283(10)$ & $0.0269(10)$ & $0.0085(8)$ & $-0.0017(8)$ & $0.0013(8)$ \\
\hline $\mathrm{C} 7$ & $0.0445(12)$ & $0.0273(10)$ & $0.0279(10)$ & $0.0052(9)$ & $-0.0016(9)$ & $0.0004(8)$ \\
\hline C8 & $0.0429(12)$ & $0.0308(11)$ & $0.0366(11)$ & $-0.0016(9)$ & $-0.0027(9)$ & $0.0076(9)$ \\
\hline C9 & $0.0381(11)$ & $0.0363(11)$ & $0.0319(11)$ & $0.0075(9)$ & $0.0010(9)$ & $0.0098(9)$ \\
\hline $\mathrm{C} 10$ & $0.0472(13)$ & $0.0451(13)$ & $0.0462(13)$ & $0.0027(10)$ & $0.0095(10)$ & $0.0167(11)$ \\
\hline C11 & $0.0553(15)$ & $0.0647(16)$ & $0.0355(12)$ & $0.0115(12)$ & $0.0179(11)$ & 0.0143 \\
\hline $\mathrm{C} 12$ & $0.0501(14)$ & $0.0526(14)$ & $0.0292(11)$ & $0.0161(11)$ & $0.0081(10)$ & $0.0029(10)$ \\
\hline $\mathrm{C} 13$ & $0.0403(12)$ & $0.0391(12)$ & $0.0247(10)$ & $0.0163(9)$ & $-0.0002(8)$ & $0.0027(8)$ \\
\hline C14 & $0.0487(13)$ & $0.0371(12)$ & $0.0285(11)$ & $0.0123(10)$ & $-0.0054(9)$ & $-0.0064(9)$ \\
\hline $\mathrm{C} 15$ & $0.0436(12)$ & $0.0320(11)$ & $0.0362(11)$ & $0.0028(9)$ & $-0.0073(9)$ & $-0.0017(9)$ \\
\hline C16 & $0.0314(11)$ & $0.0331(11)$ & $0.0301(10)$ & $0.0037(8)$ & $-0.0035(8)$ & $0.0014(8)$ \\
\hline $\mathrm{C} 17$ & $0.0362(11)$ & $0.0383(12)$ & $0.0385(11)$ & $-0.0063(9)$ & $-0.0028(9)$ & $0.0031(9)$ \\
\hline C18 & $0.0318(11)$ & $0.0506(13)$ & $0.0314(11)$ & $0.0012(9)$ & $0.0052(9)$ & $0.0036(9)$ \\
\hline C19 & $0.0308(10)$ & $0.0320(10)$ & $0.0261(10)$ & $0.0088(8)$ & $-0.0019(8)$ & $0.0032(8)$ \\
\hline $\mathrm{C} 20$ & $0.0335(11)$ & $0.0332(11)$ & $0.0247(10)$ & $0.0108(8)$ & $0.0000(8)$ & $0.0054(8)$ \\
\hline $\mathrm{C} 21$ & $0.0272(10)$ & $0.0246(9)$ & $0.0288(10)$ & $0.0024(7)$ & $0.0088(8)$ & $0.0010(8)$ \\
\hline $\mathrm{C} 22$ & $0.0364(11)$ & $0.0321(11)$ & $0.0288(10)$ & $0.0077(8)$ & $-0.0003(8)$ & $-0.0010(8)$ \\
\hline $\mathrm{C} 23$ & $0.0399(12)$ & $0.0310(11)$ & $0.0378(11)$ & $0.0002(9)$ & $0.0037(9)$ & $-0.0061(9)$ \\
\hline $\mathrm{C} 24$ & $0.0507(13)$ & $0.0260(10)$ & $0.0418(12)$ & $0.0043(9)$ & $0.0041(10)$ & $0.0038(9)$ \\
\hline $\mathrm{C} 25$ & $0.0484(13)$ & $0.0303(11)$ & $0.0342(11)$ & $0.0031(9)$ & $0.0027(9)$ & $0.0071(9)$ \\
\hline $\mathrm{C} 26$ & $0.0361(11)$ & $0.0294(10)$ & $0.0254(10)$ & $0.0012(8)$ & $0.0039(8)$ & $0.0024(8)$ \\
\hline $\mathrm{C} 27$ & $0.0291(10)$ & $0.0242(9)$ & $0.0237(9)$ & $0.0036(8)$ & $0.0084(8)$ & $-0.0034(7)$ \\
\hline $\mathrm{C} 28$ & $0.0310(10)$ & $0.0320(10)$ & $0.0238(9)$ & $0.0017(8)$ & $0.0088(8)$ & $0.0039(8)$ \\
\hline $\mathrm{C} 29$ & $0.0515(13)$ & $0.0306(11)$ & $0.0277(10)$ & $0.0058(9)$ & $0.0065(9)$ & $0.0018(8)$ \\
\hline $\mathrm{C} 30$ & $0.0814(18)$ & $0.0509(14)$ & $0.0338(12)$ & $0.0183(13)$ & $0.0249(12)$ & $0.0081(10)$ \\
\hline $\mathrm{C} 31$ & $0.0625(15)$ & $0.0533(14)$ & $0.0340(12)$ & $0.0037(11)$ & $0.0122(10)$ & $0.0188(10)$ \\
\hline $\mathrm{C} 32$ & $0.0595(15)$ & $0.0352(12)$ & $0.0458(13)$ & $0.0034(10)$ & $0.0098(11)$ & $0.0140(10)$ \\
\hline C33 & $0.0628(15)$ & $0.0336(12)$ & $0.0392(12)$ & $0.0114(10)$ & $0.0191(11)$ & $0.0050(9)$ \\
\hline
\end{tabular}


supporting information

\begin{tabular}{lllllll}
$\mathrm{N} 1$ & $0.0285(8)$ & $0.0238(8)$ & $0.0245(8)$ & $0.0048(6)$ & $0.0091(6)$ & $0.0018(6)$ \\
$\mathrm{N} 2$ & $0.0272(9)$ & $0.0360(9)$ & $0.0268(8)$ & $0.0081(7)$ & $0.0095(7)$ & $0.0061(7)$ \\
$\mathrm{O} 1$ & $0.0335(7)$ & $0.0323(7)$ & $0.0280(7)$ & $0.0041(6)$ & $0.0136(6)$ & $0.0023(6)$ \\
$\mathrm{O} 2$ & $0.0284(7)$ & $0.0413(8)$ & $0.0371(8)$ & $0.0081(6)$ & $0.0103(6)$ & $0.0075(6)$ \\
\hline
\end{tabular}

Geometric parameters $\left(A,{ }^{\circ}\right)$

\begin{tabular}{|c|c|c|c|}
\hline $\mathrm{C} 1-\mathrm{O} 1$ & $1.2329(19)$ & $\mathrm{C} 21-\mathrm{N} 1$ & $1.485(2)$ \\
\hline $\mathrm{C} 1-\mathrm{N} 1$ & $1.370(2)$ & $\mathrm{C} 21-\mathrm{C} 26$ & $1.521(2)$ \\
\hline $\mathrm{C} 1-\mathrm{C} 2$ & $1.514(2)$ & $\mathrm{C} 21-\mathrm{C} 22$ & $1.527(2)$ \\
\hline $\mathrm{C} 2-\mathrm{C} 3$ & $1.529(2)$ & $\mathrm{C} 21-\mathrm{H} 21$ & 1.0000 \\
\hline $\mathrm{C} 2-\mathrm{H} 2 \mathrm{C}$ & 0.9900 & $\mathrm{C} 22-\mathrm{C} 23$ & $1.521(3)$ \\
\hline $\mathrm{C} 2-\mathrm{H} 2 \mathrm{D}$ & 0.9900 & $\mathrm{C} 22-\mathrm{H} 22 \mathrm{~A}$ & 0.9900 \\
\hline $\mathrm{C} 3-\mathrm{C} 4$ & $1.526(3)$ & $\mathrm{C} 22-\mathrm{H} 22 \mathrm{~B}$ & 0.9900 \\
\hline $\mathrm{C} 3-\mathrm{H} 3 \mathrm{~A}$ & 0.9900 & $\mathrm{C} 23-\mathrm{C} 24$ & $1.518(3)$ \\
\hline $\mathrm{C} 3-\mathrm{H} 3 \mathrm{~B}$ & 0.9900 & $\mathrm{C} 23-\mathrm{H} 23 \mathrm{~A}$ & 0.9900 \\
\hline $\mathrm{C} 4-\mathrm{C} 5$ & $1.510(2)$ & $\mathrm{C} 23-\mathrm{H} 23 \mathrm{~B}$ & 0.9900 \\
\hline $\mathrm{C} 4-\mathrm{H} 4 \mathrm{~A}$ & 0.9900 & $\mathrm{C} 24-\mathrm{C} 25$ & $1.527(3)$ \\
\hline $\mathrm{C} 4-\mathrm{H} 4 \mathrm{~B}$ & 0.9900 & $\mathrm{C} 24-\mathrm{H} 24 \mathrm{~A}$ & 0.9900 \\
\hline $\mathrm{C} 5-\mathrm{C} 18$ & $1.388(3)$ & $\mathrm{C} 24-\mathrm{H} 24 \mathrm{~B}$ & 0.9900 \\
\hline $\mathrm{C} 5-\mathrm{C} 6$ & $1.412(2)$ & $\mathrm{C} 25-\mathrm{C} 26$ & $1.525(3)$ \\
\hline $\mathrm{C} 6-\mathrm{C} 19$ & $1.427(2)$ & $\mathrm{C} 25-\mathrm{H} 25 \mathrm{~A}$ & 0.9900 \\
\hline $\mathrm{C} 6-\mathrm{C} 7$ & $1.433(3)$ & $\mathrm{C} 25-\mathrm{H} 25 \mathrm{~B}$ & 0.9900 \\
\hline $\mathrm{C} 7-\mathrm{C} 8$ & $1.348(3)$ & $\mathrm{C} 26-\mathrm{H} 26 \mathrm{~A}$ & 0.9900 \\
\hline $\mathrm{C} 7-\mathrm{H} 7$ & 0.9500 & $\mathrm{C} 26-\mathrm{H} 26 \mathrm{~B}$ & 0.9900 \\
\hline $\mathrm{C} 8-\mathrm{C} 9$ & $1.430(3)$ & $\mathrm{C} 27-\mathrm{O} 2$ & $1.213(2)$ \\
\hline $\mathrm{C} 8-\mathrm{H} 8$ & 0.9500 & $\mathrm{C} 27-\mathrm{N} 2$ & $1.328(2)$ \\
\hline $\mathrm{C} 9-\mathrm{C} 10$ & $1.398(3)$ & $\mathrm{C} 27-\mathrm{N} 1$ & $1.447(2)$ \\
\hline $\mathrm{C} 9-\mathrm{C} 20$ & $1.419(3)$ & $\mathrm{C} 28-\mathrm{N} 2$ & $1.464(2)$ \\
\hline $\mathrm{C} 10-\mathrm{C} 11$ & $1.378(3)$ & $\mathrm{C} 28-\mathrm{C} 33$ & $1.516(3)$ \\
\hline $\mathrm{C} 10-\mathrm{H} 10$ & 0.9500 & $\mathrm{C} 28-\mathrm{C} 29$ & $1.518(2)$ \\
\hline $\mathrm{C} 11-\mathrm{C} 12$ & $1.380(3)$ & $\mathrm{C} 28-\mathrm{H} 28$ & 1.0000 \\
\hline C11-H11 & 0.9500 & $\mathrm{C} 29-\mathrm{C} 30$ & $1.525(3)$ \\
\hline $\mathrm{C} 12-\mathrm{C} 13$ & $1.394(3)$ & $\mathrm{C} 29-\mathrm{H} 29 \mathrm{~A}$ & 0.9900 \\
\hline $\mathrm{C} 12-\mathrm{H} 12$ & 0.9500 & С29- H29B & 0.9900 \\
\hline $\mathrm{C} 13-\mathrm{C} 20$ & $1.425(2)$ & $\mathrm{C} 30-\mathrm{C} 31$ & $1.520(3)$ \\
\hline $\mathrm{C} 13-\mathrm{C} 14$ & $1.432(3)$ & $\mathrm{C} 30-\mathrm{H} 30 \mathrm{~A}$ & 0.9900 \\
\hline $\mathrm{C} 14-\mathrm{C} 15$ & $1.343(3)$ & С $30-\mathrm{H} 30 \mathrm{~B}$ & 0.9900 \\
\hline C14-H14 & 0.9500 & $\mathrm{C} 31-\mathrm{C} 32$ & 1.509 (3) \\
\hline $\mathrm{C} 15-\mathrm{C} 16$ & $1.438(3)$ & $\mathrm{C} 31-\mathrm{H} 31 \mathrm{~A}$ & 0.9900 \\
\hline $\mathrm{C} 15-\mathrm{H} 15$ & 0.9500 & $\mathrm{C} 31-\mathrm{H} 31 \mathrm{~B}$ & 0.9900 \\
\hline $\mathrm{C} 16-\mathrm{C} 17$ & $1.391(3)$ & $\mathrm{C} 32-\mathrm{C} 33$ & $1.531(3)$ \\
\hline $\mathrm{C} 16-\mathrm{C} 19$ & $1.419(3)$ & $\mathrm{C} 32-\mathrm{H} 32 \mathrm{~A}$ & 0.9900 \\
\hline $\mathrm{C} 17-\mathrm{C} 18$ & $1.382(3)$ & С $32-\mathrm{H} 32 \mathrm{~B}$ & 0.9900 \\
\hline C17-H17 & 0.9500 & C $33-\mathrm{H} 33 \mathrm{~A}$ & 0.9900 \\
\hline C18-H18 & 0.9500 & С $33-\mathrm{H} 33 \mathrm{~B}$ & 0.9900 \\
\hline $\mathrm{C} 19-\mathrm{C} 20$ & $1.428(2)$ & $\mathrm{N} 2-\mathrm{H} 2 \mathrm{~A}$ & $0.855(9)$ \\
\hline
\end{tabular}




\begin{tabular}{|c|c|c|c|}
\hline $\mathrm{O} 1-\mathrm{C} 1-\mathrm{N} 1$ & $120.38(16)$ & $\mathrm{C} 23-\mathrm{C} 22-\mathrm{C} 21$ & $110.36(15)$ \\
\hline $\mathrm{O} 1-\mathrm{C} 1-\mathrm{C} 2$ & $121.25(14)$ & $\mathrm{C} 23-\mathrm{C} 22-\mathrm{H} 22 \mathrm{~A}$ & 109.6 \\
\hline $\mathrm{N} 1-\mathrm{C} 1-\mathrm{C} 2$ & $118.37(14)$ & $\mathrm{C} 21-\mathrm{C} 22-\mathrm{H} 22 \mathrm{~A}$ & 109.6 \\
\hline $\mathrm{C} 1-\mathrm{C} 2-\mathrm{C} 3$ & $112.77(14)$ & $\mathrm{C} 23-\mathrm{C} 22-\mathrm{H} 22 \mathrm{~B}$ & 109.6 \\
\hline $\mathrm{C} 1-\mathrm{C} 2-\mathrm{H} 2 \mathrm{C}$ & 109.0 & $\mathrm{C} 21-\mathrm{C} 22-\mathrm{H} 22 \mathrm{~B}$ & 109.6 \\
\hline $\mathrm{C} 3-\mathrm{C} 2-\mathrm{H} 2 \mathrm{C}$ & 109.0 & $\mathrm{H} 22 \mathrm{~A}-\mathrm{C} 22-\mathrm{H} 22 \mathrm{~B}$ & 108.1 \\
\hline $\mathrm{C} 1-\mathrm{C} 2-\mathrm{H} 2 \mathrm{D}$ & 109.0 & $\mathrm{C} 24-\mathrm{C} 23-\mathrm{C} 22$ & $111.45(16)$ \\
\hline $\mathrm{C} 3-\mathrm{C} 2-\mathrm{H} 2 \mathrm{D}$ & 109.0 & $\mathrm{C} 24-\mathrm{C} 23-\mathrm{H} 23 \mathrm{~A}$ & 109.3 \\
\hline $\mathrm{H} 2 \mathrm{C}-\mathrm{C} 2-\mathrm{H} 2 \mathrm{D}$ & 107.8 & $\mathrm{C} 22-\mathrm{C} 23-\mathrm{H} 23 \mathrm{~A}$ & 109.3 \\
\hline $\mathrm{C} 4-\mathrm{C} 3-\mathrm{C} 2$ & $112.71(14)$ & $\mathrm{C} 24-\mathrm{C} 23-\mathrm{H} 23 \mathrm{~B}$ & 109.3 \\
\hline $\mathrm{C} 4-\mathrm{C} 3-\mathrm{H} 3 \mathrm{~A}$ & 109.0 & $\mathrm{C} 22-\mathrm{C} 23-\mathrm{H} 23 \mathrm{~B}$ & 109.3 \\
\hline $\mathrm{C} 2-\mathrm{C} 3-\mathrm{H} 3 \mathrm{~A}$ & 109.0 & $\mathrm{H} 23 \mathrm{~A}-\mathrm{C} 23-\mathrm{H} 23 \mathrm{~B}$ & 108.0 \\
\hline $\mathrm{C} 4-\mathrm{C} 3-\mathrm{H} 3 \mathrm{~B}$ & 109.0 & $\mathrm{C} 23-\mathrm{C} 24-\mathrm{C} 25$ & $111.59(16)$ \\
\hline $\mathrm{C} 2-\mathrm{C} 3-\mathrm{H} 3 \mathrm{~B}$ & 109.0 & $\mathrm{C} 23-\mathrm{C} 24-\mathrm{H} 24 \mathrm{~A}$ & 109.3 \\
\hline $\mathrm{H} 3 \mathrm{~A}-\mathrm{C} 3-\mathrm{H} 3 \mathrm{~B}$ & 107.8 & $\mathrm{C} 25-\mathrm{C} 24-\mathrm{H} 24 \mathrm{~A}$ & 109.3 \\
\hline $\mathrm{C} 5-\mathrm{C} 4-\mathrm{C} 3$ & $112.66(15)$ & $\mathrm{C} 23-\mathrm{C} 24-\mathrm{H} 24 \mathrm{~B}$ & 109.3 \\
\hline $\mathrm{C} 5-\mathrm{C} 4-\mathrm{H} 4 \mathrm{~A}$ & 109.1 & $\mathrm{C} 25-\mathrm{C} 24-\mathrm{H} 24 \mathrm{~B}$ & 109.3 \\
\hline $\mathrm{C} 3-\mathrm{C} 4-\mathrm{H} 4 \mathrm{~A}$ & 109.1 & $\mathrm{H} 24 \mathrm{~A}-\mathrm{C} 24-\mathrm{H} 24 \mathrm{~B}$ & 108.0 \\
\hline $\mathrm{C} 5-\mathrm{C} 4-\mathrm{H} 4 \mathrm{~B}$ & 109.1 & $\mathrm{C} 26-\mathrm{C} 25-\mathrm{C} 24$ & $111.40(15)$ \\
\hline $\mathrm{C} 3-\mathrm{C} 4-\mathrm{H} 4 \mathrm{~B}$ & 109.1 & $\mathrm{C} 26-\mathrm{C} 25-\mathrm{H} 25 \mathrm{~A}$ & 109.3 \\
\hline $\mathrm{H} 4 \mathrm{~A}-\mathrm{C} 4-\mathrm{H} 4 \mathrm{~B}$ & 107.8 & $\mathrm{C} 24-\mathrm{C} 25-\mathrm{H} 25 \mathrm{~A}$ & 109.3 \\
\hline $\mathrm{C} 18-\mathrm{C} 5-\mathrm{C} 6$ & $118.59(16)$ & $\mathrm{C} 26-\mathrm{C} 25-\mathrm{H} 25 \mathrm{~B}$ & 109.3 \\
\hline $\mathrm{C} 18-\mathrm{C} 5-\mathrm{C} 4$ & $119.68(17)$ & $\mathrm{C} 24-\mathrm{C} 25-\mathrm{H} 25 \mathrm{~B}$ & 109.3 \\
\hline $\mathrm{C} 6-\mathrm{C} 5-\mathrm{C} 4$ & $121.66(17)$ & $\mathrm{H} 25 \mathrm{~A}-\mathrm{C} 25-\mathrm{H} 25 \mathrm{~B}$ & 108.0 \\
\hline $\mathrm{C} 5-\mathrm{C} 6-\mathrm{C} 19$ & $119.51(17)$ & $\mathrm{C} 21-\mathrm{C} 26-\mathrm{C} 25$ & $109.86(15)$ \\
\hline $\mathrm{C} 5-\mathrm{C} 6-\mathrm{C} 7$ & $122.80(16)$ & $\mathrm{C} 21-\mathrm{C} 26-\mathrm{H} 26 \mathrm{~A}$ & 109.7 \\
\hline $\mathrm{C} 19-\mathrm{C} 6-\mathrm{C} 7$ & $117.69(16)$ & $\mathrm{C} 25-\mathrm{C} 26-\mathrm{H} 26 \mathrm{~A}$ & 109.7 \\
\hline $\mathrm{C} 8-\mathrm{C} 7-\mathrm{C} 6$ & $121.81(17)$ & $\mathrm{C} 21-\mathrm{C} 26-\mathrm{H} 26 \mathrm{~B}$ & 109.7 \\
\hline $\mathrm{C} 8-\mathrm{C} 7-\mathrm{H} 7$ & 119.1 & $\mathrm{C} 25-\mathrm{C} 26-\mathrm{H} 26 \mathrm{~B}$ & 109.7 \\
\hline $\mathrm{C} 6-\mathrm{C} 7-\mathrm{H} 7$ & 119.1 & $\mathrm{H} 26 \mathrm{~A}-\mathrm{C} 26-\mathrm{H} 26 \mathrm{~B}$ & 108.2 \\
\hline $\mathrm{C} 7-\mathrm{C} 8-\mathrm{C} 9$ & $121.97(18)$ & $\mathrm{O} 2-\mathrm{C} 27-\mathrm{N} 2$ & $125.42(16)$ \\
\hline $\mathrm{C} 7-\mathrm{C} 8-\mathrm{H} 8$ & 119.0 & $\mathrm{O} 2-\mathrm{C} 27-\mathrm{N} 1$ & $120.55(16)$ \\
\hline $\mathrm{C} 9-\mathrm{C} 8-\mathrm{H} 8$ & 119.0 & $\mathrm{~N} 2-\mathrm{C} 27-\mathrm{N} 1$ & $113.96(15)$ \\
\hline $\mathrm{C} 10-\mathrm{C} 9-\mathrm{C} 20$ & $119.10(18)$ & $\mathrm{N} 2-\mathrm{C} 28-\mathrm{C} 33$ & $110.08(14)$ \\
\hline $\mathrm{C} 10-\mathrm{C} 9-\mathrm{C} 8$ & $122.84(19)$ & $\mathrm{N} 2-\mathrm{C} 28-\mathrm{C} 29$ & $111.64(15)$ \\
\hline $\mathrm{C} 20-\mathrm{C} 9-\mathrm{C} 8$ & $118.06(17)$ & $\mathrm{C} 33-\mathrm{C} 28-\mathrm{C} 29$ & $110.95(16)$ \\
\hline $\mathrm{C} 11-\mathrm{C} 10-\mathrm{C} 9$ & $120.8(2)$ & $\mathrm{N} 2-\mathrm{C} 28-\mathrm{H} 28$ & 108.0 \\
\hline $\mathrm{C} 11-\mathrm{C} 10-\mathrm{H} 10$ & 119.6 & $\mathrm{C} 33-\mathrm{C} 28-\mathrm{H} 28$ & 108.0 \\
\hline $\mathrm{C} 9-\mathrm{C} 10-\mathrm{H} 10$ & 119.6 & $\mathrm{C} 29-\mathrm{C} 28-\mathrm{H} 28$ & 108.0 \\
\hline $\mathrm{C} 10-\mathrm{C} 11-\mathrm{C} 12$ & $120.65(19)$ & $\mathrm{C} 28-\mathrm{C} 29-\mathrm{C} 30$ & $111.25(16)$ \\
\hline $\mathrm{C} 10-\mathrm{C} 11-\mathrm{H} 11$ & 119.7 & $\mathrm{C} 28-\mathrm{C} 29-\mathrm{H} 29 \mathrm{~A}$ & 109.4 \\
\hline $\mathrm{C} 12-\mathrm{C} 11-\mathrm{H} 11$ & 119.7 & $\mathrm{C} 30-\mathrm{C} 29-\mathrm{H} 29 \mathrm{~A}$ & 109.4 \\
\hline $\mathrm{C} 11-\mathrm{C} 12-\mathrm{C} 13$ & $121.16(19)$ & $\mathrm{C} 28-\mathrm{C} 29-\mathrm{H} 29 \mathrm{~B}$ & 109.4 \\
\hline $\mathrm{C} 11-\mathrm{C} 12-\mathrm{H} 12$ & 119.4 & $\mathrm{C} 30-\mathrm{C} 29-\mathrm{H} 29 \mathrm{~B}$ & 109.4 \\
\hline $\mathrm{C} 13-\mathrm{C} 12-\mathrm{H} 12$ & 119.4 & $\mathrm{H} 29 \mathrm{~A}-\mathrm{C} 29-\mathrm{H} 29 \mathrm{~B}$ & 108.0 \\
\hline $\mathrm{C} 12-\mathrm{C} 13-\mathrm{C} 20$ & $118.67(19)$ & $\mathrm{C} 31-\mathrm{C} 30-\mathrm{C} 29$ & $111.98(17)$ \\
\hline $\mathrm{C} 12-\mathrm{C} 13-\mathrm{C} 14$ & $122.89(18)$ & $\mathrm{C} 31-\mathrm{C} 30-\mathrm{H} 30 \mathrm{~A}$ & 109.2 \\
\hline $\mathrm{C} 20-\mathrm{C} 13-\mathrm{C} 14$ & $118.43(17)$ & $\mathrm{C} 29-\mathrm{C} 30-\mathrm{H} 30 \mathrm{~A}$ & 109.2 \\
\hline
\end{tabular}




$\begin{array}{ll}\mathrm{C} 15-\mathrm{C} 14-\mathrm{C} 13 & 121.50(18) \\ \mathrm{C} 15-\mathrm{C} 14-\mathrm{H} 14 & 119.2 \\ \mathrm{C} 13-\mathrm{C} 14-\mathrm{H} 14 & 119.2 \\ \mathrm{C} 14-\mathrm{C} 15-\mathrm{C} 16 & 121.61(19) \\ \mathrm{C} 14-\mathrm{C} 15-\mathrm{H} 15 & 119.2 \\ \mathrm{C} 16-\mathrm{C} 15-\mathrm{H} 15 & 119.2 \\ \mathrm{C} 17-\mathrm{C} 16-\mathrm{C} 19 & 118.72(16) \\ \mathrm{C} 17-\mathrm{C} 16-\mathrm{C} 15 & 122.57(18) \\ \mathrm{C} 19-\mathrm{C} 16-\mathrm{C} 15 & 118.71(17) \\ \mathrm{C} 18-\mathrm{C} 17-\mathrm{C} 16 & 120.66(18) \\ \mathrm{C} 18-\mathrm{C} 17-\mathrm{H} 17 & 119.7 \\ \mathrm{C} 16-\mathrm{C} 17-\mathrm{H} 17 & 119.7 \\ \mathrm{C} 17-\mathrm{C} 18-\mathrm{C} 5 & 122.37(18) \\ \mathrm{C} 17-\mathrm{C} 18-\mathrm{H} 18 & 118.8 \\ \mathrm{C} 5-\mathrm{C} 18-\mathrm{H} 18 & 118.8 \\ \mathrm{C} 16-\mathrm{C} 19-\mathrm{C} 6 & 120.14(16) \\ \mathrm{C} 16-\mathrm{C} 19-\mathrm{C} 20 & 119.56(16) \\ \mathrm{C} 6-\mathrm{C} 19-\mathrm{C} 20 & 120.30(17) \\ \mathrm{C} 9-\mathrm{C} 20-\mathrm{C} 13 & 119.65(17) \\ \mathrm{C} 9-\mathrm{C} 20-\mathrm{C} 19 & 120.16(16) \\ \mathrm{C} 13-\mathrm{C} 20-\mathrm{C} 19 & 120.18(18) \\ \mathrm{N} 1-\mathrm{C} 21-\mathrm{C} 26 & 112.10(14) \\ \mathrm{N} 1-\mathrm{C} 21-\mathrm{C} 22 & 111.76(14) \\ \mathrm{C} 26-\mathrm{C} 21-\mathrm{C} 22 & 111.18(14) \\ \mathrm{N} 1-\mathrm{C} 21-\mathrm{H} 21 & 107.2 \\ \mathrm{C} 26-\mathrm{C} 21-\mathrm{H} 21 & 107.2 \\ \mathrm{C} 22-\mathrm{C} 21-\mathrm{H} 21 & 107.2\end{array}$

$\mathrm{C} 22-\mathrm{C} 21-\mathrm{H} 21$

$\mathrm{O} 1-\mathrm{C} 1-\mathrm{C} 2-\mathrm{C} 3$

$\mathrm{N} 1-\mathrm{C} 1-\mathrm{C} 2-\mathrm{C} 3$

$\mathrm{C} 1-\mathrm{C} 2-\mathrm{C} 3-\mathrm{C} 4$

$\mathrm{C} 2-\mathrm{C} 3-\mathrm{C} 4-\mathrm{C} 5$

$\mathrm{C} 3-\mathrm{C} 4-\mathrm{C} 5-\mathrm{C} 18$

$\mathrm{C} 3-\mathrm{C} 4-\mathrm{C} 5-\mathrm{C} 6$

$\mathrm{C} 18-\mathrm{C} 5-\mathrm{C} 6-\mathrm{C} 19$

$\mathrm{C} 4-\mathrm{C} 5-\mathrm{C} 6-\mathrm{C} 19$

$\mathrm{C} 18-\mathrm{C} 5-\mathrm{C} 6-\mathrm{C} 7$

$\mathrm{C} 4-\mathrm{C} 5-\mathrm{C} 6-\mathrm{C} 7$

$\mathrm{C} 5-\mathrm{C} 6-\mathrm{C} 7-\mathrm{C} 8$

$\mathrm{C} 19-\mathrm{C} 6-\mathrm{C} 7-\mathrm{C} 8$

$\mathrm{C} 6-\mathrm{C} 7-\mathrm{C} 8-\mathrm{C} 9$

$\mathrm{C} 7-\mathrm{C} 8-\mathrm{C} 9-\mathrm{C} 10$

$\mathrm{C} 7-\mathrm{C} 8-\mathrm{C} 9-\mathrm{C} 20$

$\mathrm{C} 20-\mathrm{C} 9-\mathrm{C} 10-\mathrm{C} 11$

$\mathrm{C} 8-\mathrm{C} 9-\mathrm{C} 10-\mathrm{C} 11$

$\mathrm{C} 9-\mathrm{C} 10-\mathrm{C} 11-\mathrm{C} 12$

$\mathrm{C} 10-\mathrm{C} 11-\mathrm{C} 12-\mathrm{C} 13$

$\mathrm{C} 11-\mathrm{C} 12-\mathrm{C} 13-\mathrm{C} 20$
$\mathrm{C} 31-\mathrm{C} 30-\mathrm{H} 30 \mathrm{~B}$

C29- $\mathrm{C} 30-\mathrm{H} 30 \mathrm{~B}$

$\mathrm{H} 30 \mathrm{~A}-\mathrm{C} 30-\mathrm{H} 30 \mathrm{~B}$

$\mathrm{C} 32-\mathrm{C} 31-\mathrm{C} 30$

$\mathrm{C} 32-\mathrm{C} 31-\mathrm{H} 31 \mathrm{~A}$

$\mathrm{C} 30-\mathrm{C} 31-\mathrm{H} 31 \mathrm{~A}$

C32-C $31-\mathrm{H} 31 \mathrm{~B}$

C $30-\mathrm{C} 31-\mathrm{H} 31 \mathrm{~B}$

H31A-C $31-\mathrm{H} 31 \mathrm{~B}$

$\mathrm{C} 31-\mathrm{C} 32-\mathrm{C} 33$

$\mathrm{C} 31-\mathrm{C} 32-\mathrm{H} 32 \mathrm{~A}$

C $33-\mathrm{C} 32-\mathrm{H} 32 \mathrm{~A}$

C $31-\mathrm{C} 32-\mathrm{H} 32 \mathrm{~B}$

$\mathrm{C} 33-\mathrm{C} 32-\mathrm{H} 32 \mathrm{~B}$

$\mathrm{H} 32 \mathrm{~A}-\mathrm{C} 32-\mathrm{H} 32 \mathrm{~B}$

$\mathrm{C} 28-\mathrm{C} 33-\mathrm{C} 32$

C28-C33-H33A

C $32-\mathrm{C} 33-\mathrm{H} 33 \mathrm{~A}$

C28-C $33-\mathrm{H} 33 \mathrm{~B}$

C32-C $33-\mathrm{H} 33 \mathrm{~B}$

$\mathrm{H} 33 \mathrm{~A}-\mathrm{C} 33-\mathrm{H} 33 \mathrm{~B}$

$\mathrm{C} 1-\mathrm{N} 1-\mathrm{C} 27$

$\mathrm{C} 1-\mathrm{N} 1-\mathrm{C} 21$

$\mathrm{C} 27-\mathrm{N} 1-\mathrm{C} 21$

$\mathrm{C} 27-\mathrm{N} 2-\mathrm{C} 28$

$\mathrm{C} 27-\mathrm{N} 2-\mathrm{H} 2 \mathrm{~A}$

$\mathrm{C} 28-\mathrm{N} 2-\mathrm{H} 2 \mathrm{~A}$

C8-C9-C20-C19

$\mathrm{C} 12-\mathrm{C} 13-\mathrm{C} 20-\mathrm{C} 9$

$\mathrm{C} 14-\mathrm{C} 13-\mathrm{C} 20-\mathrm{C} 9$

$\mathrm{C} 12-\mathrm{C} 13-\mathrm{C} 20-\mathrm{C} 19$

$\mathrm{C} 14-\mathrm{C} 13-\mathrm{C} 20-\mathrm{C} 19$

$\mathrm{C} 16-\mathrm{C} 19-\mathrm{C} 20-\mathrm{C} 9$

C6-C19-C20-C9

$\mathrm{C} 16-\mathrm{C} 19-\mathrm{C} 20-\mathrm{C} 13$

$\mathrm{C} 6-\mathrm{C} 19-\mathrm{C} 20-\mathrm{C} 13$

$\mathrm{N} 1-\mathrm{C} 21-\mathrm{C} 22-\mathrm{C} 23$

$\mathrm{C} 26-\mathrm{C} 21-\mathrm{C} 22-\mathrm{C} 23$

$\mathrm{C} 21-\mathrm{C} 22-\mathrm{C} 23-\mathrm{C} 24$

$\mathrm{C} 22-\mathrm{C} 23-\mathrm{C} 24-\mathrm{C} 25$

$\mathrm{C} 23-\mathrm{C} 24-\mathrm{C} 25-\mathrm{C} 26$

$\mathrm{N} 1-\mathrm{C} 21-\mathrm{C} 26-\mathrm{C} 25$

$\mathrm{C} 22-\mathrm{C} 21-\mathrm{C} 26-\mathrm{C} 25$

$\mathrm{C} 24-\mathrm{C} 25-\mathrm{C} 26-\mathrm{C} 21$

$\mathrm{N} 2-\mathrm{C} 28-\mathrm{C} 29-\mathrm{C} 30$

$\mathrm{C} 33-\mathrm{C} 28-\mathrm{C} 29-\mathrm{C} 30$

$\mathrm{C} 28-\mathrm{C} 29-\mathrm{C} 30-\mathrm{C} 31$
109.2

109.2

107.9

111.46 (17)

109.3

109.3

109.3

109.3

108.0

110.94 (17)

109.5

109.5

109.5

109.5

108.0

111.45 (16)

109.3

109.3

109.3

109.3

108.0

123.78 (14)

119.09 (14)

$116.20(13)$

$121.70(15)$

$119.8(12)$

118.4 (12)

$-0.3(3)$

0.5 (3)

$-179.06(17)$

179.60 (16)

0.1 (3)

179.55 (16)

$-0.2(3)$

0.4 (3)

$-179.31(16)$

-176.13 (15)

$57.8(2)$

$-55.6(2)$

54.4 (2)

$-54.8(2)$

176.17 (14)

$-57.92(19)$

56.1 (2)

178.07 (16)

$54.9(2)$

$-54.2(3)$ 


\section{supporting information}

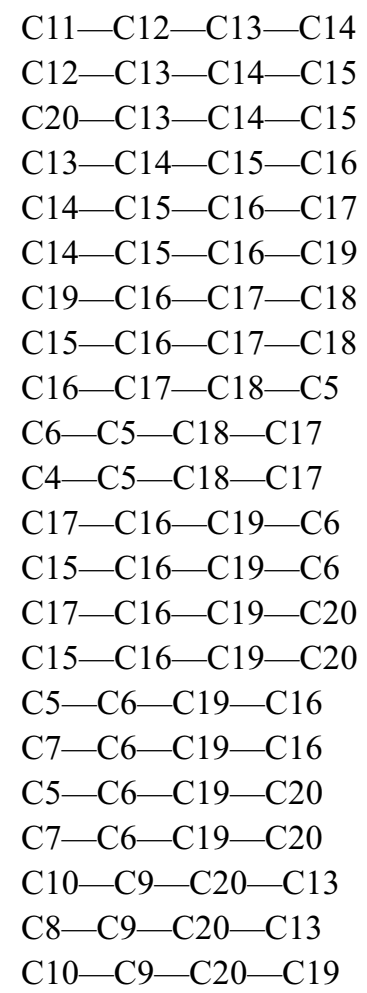

$179.83(19)$

$-179.73(19)$

-0.2 (3)

-0.1 (3)

$-179.91(18)$

$0.6(3)$

$0.2(3)$

$-179.24(18)$

$0.3(3)$

$-0.5(3)$

$176.71(18)$

$-0.5(3)$

$178.97(16)$

$179.76(17)$

-0.7 (3)

0.3 (3)

$-179.21(16)$

$-179.97(16)$

0.5 (2)

-0.7 (3)

$178.86(17)$

$-179.85(17)$
$\mathrm{C} 29-\mathrm{C} 30-\mathrm{C} 31-\mathrm{C} 32$

$\mathrm{C} 30-\mathrm{C} 31-\mathrm{C} 32-\mathrm{C} 33$

$\mathrm{N} 2-\mathrm{C} 28-\mathrm{C} 33-\mathrm{C} 32$

$\mathrm{C} 29-\mathrm{C} 28-\mathrm{C} 33-\mathrm{C} 32$

$\mathrm{C} 31-\mathrm{C} 32-\mathrm{C} 33-\mathrm{C} 28$

$\mathrm{O} 1-\mathrm{C} 1-\mathrm{N} 1-\mathrm{C} 27$

$\mathrm{C} 2-\mathrm{C} 1-\mathrm{N} 1-\mathrm{C} 27$

$\mathrm{O} 1-\mathrm{C} 1-\mathrm{N} 1-\mathrm{C} 21$

$\mathrm{C} 2-\mathrm{C} 1-\mathrm{N} 1-\mathrm{C} 21$

$\mathrm{O} 2-\mathrm{C} 27-\mathrm{N} 1-\mathrm{C} 1$

$\mathrm{N} 2-\mathrm{C} 27-\mathrm{N} 1-\mathrm{C} 1$

$\mathrm{O} 2-\mathrm{C} 27-\mathrm{N} 1-\mathrm{C} 21$

$\mathrm{N} 2-\mathrm{C} 27-\mathrm{N} 1-\mathrm{C} 21$

$\mathrm{C} 26-\mathrm{C} 21-\mathrm{N} 1-\mathrm{C} 1$

$\mathrm{C} 22-\mathrm{C} 21-\mathrm{N} 1-\mathrm{C} 1$

$\mathrm{C} 26-\mathrm{C} 21-\mathrm{N} 1-\mathrm{C} 27$

$\mathrm{C} 22-\mathrm{C} 21-\mathrm{N} 1-\mathrm{C} 27$

$\mathrm{O} 2-\mathrm{C} 27-\mathrm{N} 2-\mathrm{C} 28$

$\mathrm{N} 1-\mathrm{C} 27-\mathrm{N} 2-\mathrm{C} 28$

$\mathrm{C} 33-\mathrm{C} 28-\mathrm{N} 2-\mathrm{C} 27$

$\mathrm{C} 29-\mathrm{C} 28-\mathrm{N} 2-\mathrm{C} 27$
$54.4(3)$

$-54.9(3)$

179.84 (17)

$-56.1(2)$

$56.2(2)$

$-178.60(15)$

1.4 (2)

$-10.1(2)$

169.94 (15)

118.43 (19)

$-64.3(2)$

-50.4 (2)

126.88 (16)

$-82.44(19)$

$151.97(15)$

86.94 (18)

-38.7 (2)

$-5.7(3)$

177.15 (14)

-147.59 (18)

88.7 (2)

Hydrogen-bond geometry $\left(\AA,{ }^{\circ}\right)$

\begin{tabular}{lllll}
\hline$D-\mathrm{H} \cdots A$ & $D-\mathrm{H}$ & $\mathrm{H} \cdots A$ & $D \cdots A$ & $D-\mathrm{H} \cdots A$ \\
\hline $\mathrm{N} 2-\mathrm{H} 2 A \cdots \mathrm{O} 1^{\mathrm{i}}$ & $0.86(1)$ & $2.17(1)$ & $3.026(2)$ & $176(2)$ \\
$\mathrm{C} 2-\mathrm{H} 2 D \cdots \mathrm{O} 2^{\mathrm{ii}}$ & 0.99 & 2.49 & $3.358(2)$ & 146 \\
$\mathrm{C} 4-\mathrm{H} 4 B \cdots \mathrm{O} 2^{\mathrm{ii}}$ & 0.99 & 2.45 & $3.302(2)$ & 144 \\
\hline
\end{tabular}

Symmetry codes: (i) $-x,-y+1,-z+1$; (ii) $-x+1,-y+1,-z+1$. 